\title{
Dyslexia: A Review about a Disorder That Still Needs New Approaches and a Creative Education
}

\author{
Lucianne Fragel-Madeira' ${ }^{1,2^{*}}$, Juliana S. C. de Castro" ${ }^{1 \#, ~ C r i s t i n a ~ M . ~ C . ~ D e l o u ~}{ }^{1,2^{*}}$, \\ Waisenhowerk V. Melo', Gustavo Henrique V. S. Alves ${ }^{1}$, Patricia P. Teixeira1, \\ Helena C. Castro1,2 \\ ${ }^{1}$ Post Graduate Program in Science and Biotechnology (PPBI), Federal Fluminense University, Niterói, Brazil \\ ${ }^{2}$ Post Graduate Program in Diversity and Inclusion (CMPDI), Federal Fluminense University, Niterói, Brazil \\ Email: ${ }^{\text {Ifragel@id.uff.br, }}$ ㄷistinadelou@globo.com
}

Received 18 October 2014; accepted 23 June 2015; published 26 June 2015

Copyright (C) 2015 by authors and Scientific Research Publishing Inc.

This work is licensed under the Creative Commons Attribution International License (CC BY).

http://creativecommons.org/licenses/by/4.0/

(c) (i) Open Access

\begin{abstract}
Dyslexia is a neurological disorder of genetic origin that affects reading. Importantly, the scholar performance of the dyslexic students depends on several aspects, including the educational system and the teaching-learning process used, and the relationship with family members, teachers and other students. Thus, it is necessary to inform about approaching the issues related to dyslexia and the education of the affected people. In addition, we should raise the scientific questions about the need of this public of a greater investment on research and professional formation. In this paper, we reviewed some specific topics of dyslexia, such as the neurological profile and the educational needs of those affected by this disorder to clarify the issues that surround and complicate the process of teaching and learning of these individuals that still need help and a creative education.
\end{abstract}

\section{Keywords}

Dyslexia, Inclusive Education, Reading, Teaching-Learning Process

\section{Introduction}

Developmental dyslexia is a neurological disorder of genetic origin that specifically affects reading (Eicher et al.,

*Corresponding authors.

"Both authors contribute equally to the work.

How to cite this paper: Fragel-Madeira, L. et al. (2015). Dyslexia: A Review about a Disorder That Still Needs New Approaches and a Creative Education. Creative Education, 6, 1178-1192. http://dx.doi.org/10.4236/ce.2015.611116 
2014). Importantly, the lack of accuracy or fluency in the reading process observed in dyslexic children is not due to lower intellectual or sensory impairments or to pedagogical, emotional or social-cultural problems (Kajihara, 2008; Gabrieli, 2009).

The brain of people with developmental dyslexia is different in several aspects. Scientists have detected anatomical differences as well as cellular and connect differences using different techniques. In fact, there are also variations even among the brains of dyslexic individuals (Kajihara, 2008, Leonard \& Eckert, 2008; Krafnick et al., 2014; Zdrojewska \& Coffey, 2014). Due to these anatomical and neurophysiological differences, people with dyslexia have great difficulty with written language resulting in embarrassment and learning disabilities and often drop out of school.

Although dyslexia has no cure, the good performance of these students is feasible and depends on the educational system and the teaching-learning process, besides the relationship with family members, teachers and other students (Souza, 2009). The difficulty in assimilating the written content compromises the student learning and progress in the schooling process.

To help this public, it is necessary to identify not only the issues related to this neurological disorder, but also the scientific questions that help in establishing the need for greater investment in science and health education research. Thus, the purpose of this article is to review the neurological basis of developmental dyslexia and the difficulties involved in the process of teaching the affected public.

\section{Methodology}

In order to review the topics involving dyslexia, we performed a systematic review and searched the electronic databases PubMed, SciELO, EMBASE, the Cochrane Library, and LILACS. Furthermore, we searched the internet and explored references from textbooks and reviews using the following uniterms: dyslexia, learning, Neuro, disorder, teaching.

\section{Results and Discussion}

\subsection{The Learning Process of Reading}

Learning how to read and write requires identification, recognition and understanding of words in the attempt to interpret a given text (Torres \& Fernandes, 2001; Barton et a1., 2014). The understanding of the neural processes in normal individuals assists the identification of abnormal development patterns during childhood. Students (affected or not) may serve in identifying several disorders related to the nervous system, such as dyslexia (Marsh et al., 2008). Therefore, to better understand this disorder, it is necessary to first understand the learning cognitive process.

Learning is a process by which each individual experience changes the nervous system and his/her behavior, which modulate the way how the individual act, think and feel. This happens through reorganizations in the neural systems that are responsible for perception and movement control, among others (Carlson, 2002).

According to some authors, learning is the process of changing the behavior of an individual considering three fundamental elements: the stimulatory situation, the person who learns and the response. There are several studies and theories about dyslexia and learning from different areas and in this work we will address the neurophysiologic perspective of learning.

\subsection{Neurophysiology of Learning}

Since birth or even before, mosaic of neural connections transforms new information into a new skill. The individual receives a stimulus that causes a change in his/her neural circuitry, leading to synaptic plasticity due to changes in the structure and/or in the synapses biochemistry. These modifications can follow different routes according to each individual need.

According to the literature, learning is intimately linked to changes in our behavior. These changes are called memory. Therefore, when a stimulus occurs, we use the situation and the learning previously acquired to generate an appropriate behavior in that specific moment. The memory related to this learning (positive or negative) will lead the individual to have a certain conduct when facing this situation again. The processes of learning and memory are a large sequence of steps that go from stimulus presentation until its usage. The stimulus can be an external and/or an internal event during acquisition, and the information has three destinations: a) the temporary 
retention for later use, generating an specific behavior; b) the forgetfulness, in which the stimulus information is no longer used; c) the consolidation, in which the individual retains the new information for a long time causing changes in the cerebral connections and may use this information to generate a behavior when appropriate by using the memory.

There are several anatomical structures in the brain involved in learning processes, including the associative cortices located in the frontal, temporal, occipital and parietal lobes. They were subdivided depending on the given stimulus, such as the visual association cortex and the auditory cortex of association. Another structure involved in this process is the amygdala, which organizes and standardizes the information, acting as a bridge between the perception of new a stimuli and the memorization (Carlson, 2002). The several stimuli and different results involving different pathways of neural connections led to some classifications in learning processes.

According to neurophysiology, there are four categories of learning: Perceptual Learning; Stimulus-Response Learning; Motor Learning; Relational Learning.

\subsection{Perceptual Learning}

In perceptual learning, the individual learns to recognize the stimuli that have been previously perceived, through changes in the sensory associative cortex. A good example is the recognition of images and sound that will occur through the visual associative cortex and in the auditory associative cortex, respectively.

Some studies showed a reorganization of the neural circuits in the inferior temporal cortex when a new stimuli is presented. Perceptual learning is facilitated through the activation of the central nucleus of the amygdala, which stimulates the release of acetylcholine in the cerebral cortex. This facilitates the formation of neural circuits through long-term potentiating. Therefore, the perceptual learning allows the individual to recognize certain stimuli and respond appropriately to them. However, it is not useful if it is not considered together with other forms of learning.

\subsection{Stimulus-Response Learning}

The stimulus-response learning consists in the capacity of learning to perform a particular behavior when facing a particular stimulus of two main categories: classical conditioning and operant conditioning.

\subsubsection{Classical Conditioning}

The classical conditioning involves the association between two stimuli. A very effective stimulus may modify the behavior associated with another stimulus. The stimulus that normally causes the response is called the unconditioned stimulus or unconditioned response as they do not need any prior training stimulus that normally does not evoke this response. The conditioned stimulus or conditioned response requires prior training for that particular response.

The central nucleus of the amygdala is involved in these two stimuli and plays an important role in the organization and standardization of emotional responses. The information of the two stimuli is paired and converges in the medial region of the medial geniculate nucleus (MGN) and also to the lateral amygdala that is connected to the central nucleus, responsible for emotional responses.

\subsubsection{Operant Conditioning}

Learning from the operant conditioning stimuli occurs involves a stimulus and a response. The individual learns to associate a given stimulus to a specific response, usually a motor action. This conditioning is, in most cases, associated with a reward. Thus, operant conditioning is closely related to motor learning and is the means by which most of the animals benefit from their own life experiences. As favorable responses are carried out, providing also favorable experiences, there is always a tendency to present these same responses. Thus, the neural circuits between these stimuli and these favorable responses are always strengthened.

In the operant conditioning, there is a connection between the stimulus in the environment and generally the motor response. This reveals the neural systems involved in these actions, including: the sensory cortex, where perception occurs and the motor cortex that controls movement. Direct transcortical connections and connections via basal nuclei and thalamus act on the connection between associative sensory cortex and associative motor cortex. 


\subsection{Motor Learning}

The motor learning is related to changes in the motor systems. However, these changes only occur if there are guidelines from the sensory environment. Motor learning is strongly linked to stimulus-response learning.

Both the motor prefrontal cortex and the associative sensory cortex are involved in motor responses after the perception of environmental stimuli. These are involved not only in learning, but especially in learning of automatic and routine behaviors, by sending sensory information and the planning of the movements from the neocortex to this region.

\subsection{Relational Learning}

This learning is based on individual stimuli that involve connections between different areas of the associative cortex (the associative somatosensory cortex and the auditory cortex).

Researches on relational learning show an even closer integration with the memory processes, including recovery of memories of events and episodes. Relational learning involves several types of learning such as spatial learning with the perception of spatial location and observational learning through imitation and observation of other people and situations. Although learning can be classified in different ways, it may involve all these categories, forming a large network of synapses and neural changes in brain circuits.

\section{The Language}

Communication between animals is a universal characteristic. All animals interact with the environment and with other animals by establishing communication. However, only humans use speech and writing to communicate. In men, written and spoken language are not the only from interaction, also including hand and facial gestures, and sign language, for example. Therefore, human communication is much more than only to speak, listen, read or write.

The language involving speech and writing has been widely studied throughout history. According to Kandel et al. (2002), the language is the ability to encode ideas, expressions and feelings to signals for communicating with each other. In that context, someone expresses something to another who comprehends it (Lent, 2004). The coded language comes to the brain through the visual and/or auditory systems, while the motor system produces spoken, signaled and/or written representation of the information (Bear et al., 2008). The language seems to be a very complex and unique adaptation of our species. It allows spreading culture, history, everyday life, science through the generations.

Many researches try to explain language acquisition. The behavioral approach supports language as an activity planned and taught as behavioral conditioning stimulus-response learning. Chomsky defends the genes as a determinant for the oral language where the individual is born with the ability to speak (Lent, 2004). There are other hypotheses of different areas such as the constructive view of Piaget and Vygotsky's socio-interactionist perspective where language is a genetic predisposition, but the linguistic behavior is a result from the cognitive development and of a social and cultural content, which is crucial in this event (Stefanini \& Cruz, 2006; Vygotsky, 2008). Some authors believe that all hypotheses together will be more plausible to explain this language development (Kandel et al., 2002).

According to the literature, there is a neurobiological basis of speech strongly attributed to the innate structure of the human being. In the first months after birth, a child can babble the first words through imitation of sounds and facial movements. However, the writing ability seems to be more complex and less linked to the innate structures. Apparently this learning is dependent on a formal, longer and more laborious approach. Thus, the language cannot be attributed only to a structure only inherent to learning (Kandel et al., 2002).

The knowledge about the neurophysiology of the language is recent and was only possible through studies of patients with disorders of speech and writing (Carlson, 2002; Kandel et al., 2002; Tailby et al., 2014). This topic should be somehow understood in order to allow the approach of dyslexia issues.

\subsection{Language Neurophysiology}

According to the recent studies, speaking is a lateralized behavior (Corballis, 2014). The language is largely dependent on the left hemisphere in most individuals. Most of the right-handed population has the left hemisphere in dominance for speech as well as most of left-handed individuals, even those who use non-spoken language 
(e.g. Sign Language users) and rely on visuo-spatial and motor information (e.g. deaf people). Thus, the left hemisphere is dominant in the language system as it is significantly involved in this task. Speaking is not simple, but a complex integration of ideas and thoughts that involves the formulation of phrases by using words and/or signs and symbols. Therefore the right hemisphere is also necessary for this large network of neural circuits involved in this complex task.

Two cortical areas are closely associated with the neural circuits responsible for language: Broca's area in the lateral frontal region, and Wernicke's area in the posterior superior temporal lobe, both present in the left hemisphere. According to the authors, although these terms are still widely used, the anatomical limits between these areas are not clearly defined and appear to vary from one person to another.

The main cortical area responsible for speech is Broca's area. It is related to the ability of speaking not only due to the control of the motor cortex associated with the muscles (muscles from face, tongue, larynx, etc.), but also with the ability of expressing ideas and emotions.

The Wernicke's area is responsible for decoding auditory linguistic function, identifying the sounds of words, interpreting and capturing memories of the events that are denoted by each word (Carlson, 2002; Lent, 2004; Kandel et al., 2002).

Although the right hemisphere is not dominant in the language system, it is involved in this process. Recent research in this area shows that the right hemisphere allows the understanding of numbers, letters and small words, if the response is not verbalized. Studies show that patients with hemispheres separated by surgery can function in independent ways and have different capacities for language (Bear et al., 2008).

\subsection{Neurophysiology of Reading and Writing Processes}

The communication systems include reading and writing process as complementing forms for oral communication/verbalization. There are many techniques and possibilities of writing developed by humanity. According to different authors, learning the written symbols of a particular culture emphasizes the ability of human communicative interaction (Carlson, 2002; Lent, 2004).

Writing is a great challenge for an individual as it involves various neurological activities as the formulation of ideas, the connection of words and phrases to the thoughts, the formulation of a mental image of a desired written sequence, the use of spelling rules and also controlling muscles of the hand and fingers.

The pre-motor cortex, located in front of the motor cortex of the hand, is responsible for the organization of the movement to write in the situations such as dictation, copying and development of texts. These processes are modulated by the parietal areas of sensory integration where there is a convergence of visual and auditory systems. This convergence is intended to facilitate the hand control on writing, during dictation or copying. The functional organization for writing varies greatly between one individual to another depending on how literacy was performed.

The specialization of the hemisphere determines the characteristics of the neural system involved in reading. The words found in the left visual hemisphere would be identified by the right hemisphere, being processed in the left hemisphere. However, the analysis of spatial relationships shared by the different words is best analyzed by the right hemisphere. The results of these analyses go from one hemisphere to another through the posterior portions of the corpus callosum. This creates a network connection among the words, their spatial forms, their meanings and context present in a particular reading.

Despite the left hemisphere is considered dominant for language functioning, recent studies suggest that the right hemisphere can occasionally be able to "write" or even "talk", indicating that there is not only a single brain system in a single hemisphere responsible for all aspects of the language.

There is no doubt that the eye and vision processing in primary visual cortex and extra striatum cortical areas are necessary to decode the writing and reading (Turkeltaub et al., 2014). Reading requires efficient visual skills, including the ability to process the spatial location of the letters while the eyes move across the text. These processes must be coordinated with the perceptual aspects of vision and memory, which should match the level of word decoding and linguistic processes. To provide reliable information, this must occur with a precise timing (Gabrieli, 2009).

By analyzing the concepts of learning and language together, it is possible to understand that language is much more than just a system of sounds and words. Learning a language depends on all different types of learning (e.g. perceptual, motor and relational). The individual matures its own linguistic systems and comes to do- 
minate the various processes of human communication during the use of these types of learning. New techniques for the study of different language systems pointed that the language areas are much more complex and extensive than has been suggested so far.

The language learning process is a complex system of observations, perceptions and neural connections and is related to several disorders. Recent research has classified these disorders into two major groups, according to the hemisphere that is affected, including: a) verbal learning disabilities and/or learning disorders mediated by the left brain. It is related to the compromising of language skills needed for reading, spelling or writing, and b) learning disabilities and/or nonverbal disorders mediated by the right hemisphere related to disturbances in tactile perception, psychomotor coordination, visuospatial organization, concept formation and social adaptation (Ciasca, 2004).

Among the several oral learning difficulties such as aphasia is the dyslexia. It is one of the causes of so-called "functional illiteracy" that still involves ignorance and lack of information for most population. Because of this ignorance, dyslexia is often not considered as a cause of failure and drop out by schools and/or teachers.

\section{Dyslexia}

The etymology of the word dyslexia comes from Greek DYS-disorder and LEXIA-language (Amorim, 2008). More specifically, dyslexia means difficulty in speech. However, most authors consider that this term encompasses a difficulty in learning to read (Torres \& Fernandez, 2001). Therefore, dyslexia means bad reading, being a specific difficulty in the acquisition of reading (decoding). The difficulty in reading is one of the major factors of school failure and social exclusion. Individuals unable to decode and interpret the verbal symbols in writing are isolated from the society. Reading is not an isolated skill, but part of a complex linguistic activity related to several functions. Thus, the inability to perform writing shows a deficiency in the language structure and/or general organization (Luz, 2008).

Reading requires neurological, psychological, social and environmental processes to be effective. Some of these aspects can change the process of developing the ability to read. Dyslexia is a disorder involving spoken language aphasia due to lesions in the regions actually involved in the linguistic processing (Lent, 2004). It is known as alexia, loss of ability to read without losing the ability to write, produced by brain damage (Carlson, 2002). It is independent of conventional education, but depends on the cognitive difficulties from structural and/ or functional origins.

Dyslexia involves the decrease of word acquisition and identification (Ciasca, 2004). Perea \& Panadero (2014) showed that the visual recognition of words is consistent with current models. Individuals with this disorder do not have inferior intelligence, physical, emotional, social, or any problem related to socioeconomic issues (Torres \& Fernandez, 2001). Dyslexia can be classified into two major groups: a) the acquired dyslexia, caused by brain damage after trauma or brain injury in people who already had learned to read, and b) the developmental dyslexia involving reading difficulties that become apparent when children are learning to read (Carlson, 2002).

\subsection{Origin of Dyslexia}

The scientific studies reporting dyslexia as a neurobiological disorder dated from the nineteenth century. During 1870 and 1890, some researchers, especially ophthalmologists, explored the inability of these individuals in perform independent reading (Kajihara, 2008).

The English physician Pringle Morgan in the 1890s, described a 14 year old boy that could not read despite his intellectual capacity. He had a good performance in other areas (solving mathematical equations) whereas displayed an important deficit on reading skills (Pringle-Morgan, 1896). Morgan was among the first to describe this disorder as "congenital word blindness". This was one of the first considerations about the "verbal blindness" as a development disorder occurring in healthy children despite previous observation about intelligent adult men and women, with good vision and inability to read as a result of damage brain, was reported centuries earlier. The ophthalmologist Hinshelwood observed the disorder in a family with four people with the dyslexia presented as an inherited tendency (Kajihara, 2004).

During the twentieth century, there are few studies about the neurobiology of dyslexia. Only after the instrumental technological development in the 1990s, including the functional neuroimaging techniques, it was possible to understand the cognitive functions related to speech and consequently to the dyslexia (Carlson, 2002; Kajihara, 2004). 
According to Teles (2004), the pedagogical approach in the 60s correlated dyslexia with emotional problems, and emotional immaturity, reducing the importance of the biological aspects of this disorder to the teaching and learning process of reading. Only in this decade that the term dyslexia was defined by the World Federation of Neurology, characterizing this disorder as a number of learning issues and problems involving reading even in children with proper social-cultural stimulation and normal intelligence. During the 1990s, other topics were discussed about dyslexia, in which some were not defended by doctors or researchers, but by teachers and educators. Therefore dyslexia had several names, meanings, concepts in an attempt to better explain this disability (Teles, 2004).

According to the Diagnostic and Statistical Manual Mental Disorders, DSM IV, dyslexia is a "Disruption of Reading and Writing" that follow some diagnostic criteria, including a) low performance in reading and writing compared to the expected level for the given age, b) low performance at school and/or daily activities related to reading and writing skills, and c) a worse sensory deficit in a reading activity that normally would be carried out without effort. Currently the International Association of Dyslexia adopts the following definition: Dyslexia is characterized by difficulties of neurobiological origin in the recognition of specific words, decoding and spelling. These difficulties typically result from a deficit in the phonological component of language that is often unexpected in relation to other cognitive abilities. Secondary consequences may include problems in reading comprehension and reduced reading skill that can prevent the increase in vocabulary and knowledge development.

Presently, the research related not only to dyslexia but also with many other learning disabilities is of interest not only for medical but also for educational area since the better the understanding of these disorders, the higher the possibility of improving the quality of life of the affected individuals.

\subsection{Neurophysiologic Perspective of Developmental Dyslexia}

Neurological factors responsible for a brain dysfunction, as neuronal migration (Zdrojewska \& Coffey, 2014) and biological factors (genetic changes) are consistently related to developmental dyslexia (Gabrieli, 2009). Despite that, patients with developmental dyslexia do not exhibit any decrease in other neural functions. Actually, according to Gabrieli (2009), IQ tests performed in dyslexics show that the presence of dyslexia is independent of other talents, which allows children with dyslexia to become successful adults, especially if there is monitoring of the disorder from its earliest manifestations.

The analysis of the current literature revealed that there are different perspectives for approaching dyslexia. In one hand, educators and educational psychologists try to understand the cognitive aspects of this disorder that affect the dyslexics learning. On the other hand, by using the technological advances, scientists try to discover the neurophysiologic and neuroanatomy aspects related to this disorder. Currently there is one factor in common between them, which is the fact that dyslexic individuals have differences in the brain compared to non-dyslexics (Leong \& Goswami, 2014).

Some authors describe that the emergence of developmental dyslexia has a strong hereditary influence. A child whose parents are affected by dyslexia presents a risk of $40 \%$ to $60 \%$ of developing the disease. These risks are further increased when other family members are also affected (Schumacher et al., 2007). Studies showed the occurrence of dyslexia in both twins in $68 \%$ of cases and, among those tested, $50 \%$ have a family with dyslexics. This can be considered as a fundamental characteristic for prior monitoring children who have relatives with this disorder (Gabrieli, 2009). Individuals with specific language impairment and dyslexia may exhibit aneuploidy of sex chromosome (Simpson et al., 2014).

There are several causes for dyslexia that may vary in each child. Children with late psycholinguistic and visual perceptual maturity may have specific difficulties in reading and writing because of factors such as: a) delays on the evolution and function of the left hemisphere, which produce changes in perceptual symbolic processes, and b) delays in the development of the left hemisphere caused by neuroanatomical abnormalities such as malformations of neuronal tissue (Torres \& Fernández, 2001). Thus, it is clear that developmental dyslexia is a developmental disorder and not a pathological one, since the delay in brain maturation would delay the acquisition of certain skills but not causing loss, deficit or disability.

Dyslexic individuals have a reduced vocabulary, lower fluency in verbal descriptions, using low complex sentences. Compared with non-dyslexics, these individuals fail to detect sound of letters and graphemes, which explain the problems in both reading and writing. The real problem is not related to verbal processing as dyslexics are able to understand and use language. The problem is in the phonological encoding skill, since tasks such 
as spelling, reading and writing become extremely complex for these readers. According to the literature, dyslexic children are slow in reading with a lack of pace, confusion with the letter's distribution and order, misreading of letters and words, blending sounds and phonological reading disability. In writing, the motor act is affected, causing poor handwriting, with poorly differentiated graphic letters. The spelling is also affected by the deficient perceptions and low visual memory (Torres \& Fernandez, 2001).

Through molecular and genetic studies involving families with dyslexia, mainly twins, chromosomal regions have been identified, indicating the presence of certain genes that appear to be involved in the susceptibility of the disorder (Williams et al., 2006; Bishop, 2009). However, how many genes are related to disturbance is not yet known (Svidnicki et al., 2013). These genetic studies may be potentially useful for better understanding of the neurophysiologic and cognitive processes related to this disorder (Schumacher et al., 2007).

Studies related to twins families and their genotypes have already demonstrated the possible correlation of specific genes not only with developmental dyslexia as a whole, but with the specific features of this disorder. According to genetic researches, chromosomes 6 and 15 may contain genes responsible for different components of this disorder (Carlson, 2002). In the last years a translocation of the gene DYX1C1 of chromosome 15 was identified, which seems to disrupt the function of the protein produced by this gene (Kajihara, 2008).

According to Bishop (2009), when the first genetic studies about dyslexia revealed the high character of heredity, many scientists anticipated that future genetic tests would identify dyslexia even before the start of schooling. However, genetic and neurological studies are increasingly demonstrating the complexity of this system, making this goal more distant. Therefore, it is clear that these studies are just beginning, and because of dyslexia is not a disorder with only one feature, but rather a set of factors that characterize when they met a dyslexic person, we still need a better understanding of the classification criteria of this disorder for molecular and genetic research advances.

Earlier studies on dyslexia suggested differences based on gender. In fact, it is possible to observe a higher proportion of affected males than females (Schumacher et al., 2007). The gender-related hypothesis pointed the testosterone as an important factor for the development of dyslexia. However, recent studies have deconstructed this hypothesis, since mostly male behavior in school is more dispersed than females. So teachers probably are more attentive to the development of male children than female (Salles et al., 2004).

Since the advance of technology, new studies, procedures and equipment can be used to obtain more accurate results comparing the neural circuits involved in dyslexia. According to Marsh et al. (2009), the onset of brain development occurs through a sequence of major events, starting with the formation of the neural tube and ending with myelination. The development of the brain at a macroscopic level typically proceeds first to the sensorimotor areas, spreading later to other areas throughout childhood and adolescence. Disorders in these developmental patterns appear to be involved in the pathogenesis of various diseases such as infantile dyslexia development.

According to the authors, studies with new techniques such as functional magnetic resonance imaging (fMRI) and Positron Emission Tomography (PET) allows the exploration of the relationship between brain structure and function. Cognitive processes such as language are more likely to lead to differences in brain activation patterns in children compared with adults. Probably because these brain regions are important for these functions, which suffer neuroanatomical changes during adolescence. Thus, studies involving the comparison of structural and functional development of the brain during the ripening can provide crucial information for generating hypotheses about the neural bases related to the possible development of psychopathology. Delineate the trajectory of the brain associated with improvement in reading skills during the development of the individual can serve as a reference identifying the breaks in the process contributing to a better understanding of reading in dyslexic children (Marsh et al., 2009).

Some laboratories are studying the neuroanatomical and neurophysiologic perspectives of dyslexia in search for markers that aid the development of a neurobiological classification. In fact, dyslexic individuals differ in relation to non-dyslexic individuals, but these are not well defined yet, which prevents the safe classification of these subjects (Leonard \& Eckert, 2008).

The temporal plane (planum temporale) is a region located in the temporal lobe of both hemispheres. The left planum temporale is located in the region of Wernicke. As already mentioned in previous sections of this paper, this region is related to phonological processing and, more specifically, the understanding of speech and writing. According to anatomical studies on the asymmetry of left and right temporal planes dating from the 1970 s, the 
sizes of the time plans are asymmetrical, being greater the planum temporale of the left hemisphere-dominant for language.

Research conducted with groups of dyslexic readers showed they have reversed this asymmetry or temporal planes with the same size (symmetrical). In fact, among the non-dyslexic, $70 \%$ have plans temporal asymmetric, with left side greater than right side. However, among the dyslexic, only about $30 \%$ have such an asymmetry. The size of the temporal planes is defined during pregnancy, indicating that this is a feature of congenital and may be related to genetic influences or trauma. The symmetry of the planum temporale is not a dyslexia diagnosis factor, as some non-dyslexic subjects also show this pattern. However, it is interesting to notice that the symmetry of temporal planes in dyslexic individuals is correlated with phonological processing disorders (Hynd, 1989; Etchepareborda \& Habib, 2001; Hiemenz \& Hynd, 1997; Machado et al., 2006; Leonard \& Eckert, 2008).

Functional neuroimaging studies have revealed differences in brain function of dyslexic individuals. There are specific patterns of brain activation, atypical in the presence of dyslexia during processes of language, such as reading. When performing a task that requires phonological awareness for printing (writing) such as how to decide whether to use certain letter, word, rhyme etc, the temporo-parietal cortex of the individual is recruited regardless of age. In contrast, children and adults with dyslexia show reduced or absent activation in this region. As demonstrated in some studies, the left parietal-temporal region is correlated with auditory and visual processes during reading. Unlike fluent readers, dyslexic readers did not show increased activation in this region which is increased in the phonological analysis during a task. Furthermore, readers dyslexic also present activation in the opposite region with a greater temporal activation of the right posterior region (Gabrieli cited Rumsey, 2009; Temple, 2001).

An interesting observation is the similarity of these neural differences across people from different cultures. However, neuroimaging studies revealed cultural influences in the activation of dyslexic according to the nationality (including hipoactivation). Interestingly, there are differences in neural activations among people of French, Italian, English, Chinese, etc. In addition, reading ability is correlated with individual differences and their responses to visual stimuli. Thus, not only dyslexia is associated with other factors, but also involves specific characteristics of each individual. According to Gabrieli (2001), the use of new technologies has allowed the identification of new features found in various types of readers, whether dyslexic or not. Through a magnetic resonance diffusion tensor images (DTI) we may identify the white matter, which provides a quantitative index of the organization of large myelinized axons. Thus, the DTI performance of readers with dyslexic shows that the substance white appears lower in the left posterior cerebral region than in non-dyslexic readers. Therefore, this measure is probably related to the central cores of reading.

More specific studies have shown that dyslexia may be involved in a brain area even more, being a consequence of disturbances in the development and functioning of the left hemisphere, specifically in the parietal and occipital-temporal (Marsh et al., 2009). Studies conducted with dyslexic and non-dyslexic individuals showed that dyslexic depends increasingly on the posterior portion of the left temporal-occipital region with increasing age, whereas normal subjects rely more on the anterior temporal-occipital region of the left hemisphere over the years. These results suggest that dyslexic children have a posterior neural system when mature. In contrast, nondyslexic readers increasingly depend on an anterior region with advancing age. Although more research is needed, these studies suggest that dyslexia may result from a functional disorder early this anterior temporaloccipital region, known as the training area of the word, which in adult non-dyslexic readers supports the development of skills related to reading. This fact justifies the fact that some dyslexic children have difficulties at the junction of letters and word formation, which influences in a difficulty of textual interpretation.

Another field of neurological research investigates the various regions and neural connections involved in dyslexia looking into the cerebellum. The research conducted in the last 20 years, raised the question of children's deficits in tasks of fast naming pictures, balance, fine motor control and language skills that may be related to the perfect functioning of the cerebellum (Facoetti et al., 2001; Eckert et al., 2003).

The cerebellum is a brain structure involved in the process by which the motor tasks become automated. Deficiencies in the execution of motor skills in automation and speed of information processing, are present in children with dyslexia, pointing to the involvement of cerebellar dysfunction (Facoetti et al., 2001; Eckert et al., 2003; Etchepareborda \& Habib, 2001). The author's research has demonstrated the role of the cerebellum in the phonological issue, assuming that cerebellar function may be connected to the recovery of the words, common mistake of dyslexic children. Research conducted in children with tumors in the cerebellum demonstrates a role of the left hemisphere cerebellar central to the process language (Eckert et al., 2003). 
Neuroimaging studies have shown that the cerebellum is not only involved in an abnormal metabolism in the right lobe of the cerebellum of dyslexic readers but also in the smaller size, as confirmed by the studies of Eckert et al. (2003). The cerebellum was measured to identify differences between individuals and a decrease was detected in the cerebellum of dyslexic compared to non-dyslexic. Some scientists still do not claim that there is a connection between these differences and characteristics displayed by dyslexics, but that there is no relationship between dyslexia and cerebellar dysfunction.

One of the most debated theories, discussed and researched in relation to learning disabilities with a focus on dyslexia seems to be the magnocellular theory, which proposes that the cause for the reading disorder is a loss in giant cells that form the visual processing pathway that extends from the retina to the brain (Stein \& Walsh, 1997; Talcott, 1998).

The visual system has two main components, the magnocellular and parvocellular system, named for the two types of layers in the lateral geniculate nucleus, which is the gateway to the visual cortex, and thus for the visual perception conscious. The parvocellular system is projected to the visual cortex and then to the temporal cortex, which is where the object happens to be identified based on color, shape and texture. The magnocellular system is distributed widely across the retina and protrudes through the ventral lateral geniculate nucleus to the visual cortex and then largely into the parietal cortex, with neurons with large cell bodies that convey information about movement, depth, and small differences contrast. The magnocellular system ends in the posterior parietal cortex and has an important role in visual attention, attention, visuo-spatial and peripheral vision and these are essential components of effective reading (Bear et al., 2008; Carlson, 2002).

The possibility that abnormalities in the functioning of the magnocellular visual system are involved in the genesis of dyslexia has been reviewed by different authors. According to Kajihara (2008), the layers of magnocellular dyslexic individuals are disorganized with smaller cell bodies. The cell bodies were $27 \%$ lower and more variable both in size and shape, while the parvocellular layers appear to be equal to the layers of non-dyslexic individuals. Through experiments and research conducted by Stein \& Walsh (1997) and Talcott (1998) noted that the primary target of the magnocellular system is the posterior parietal lobe, the terminal point of the dorsal visual system. This route is related to the "where" vision, e.g. (so far), on information regarding the location of the object (Carlson, 2002).

The researchers point out a number of difficulties for dyslexics including problems with the perception of space and movement, lack of coordination, difficulty with balance, poor handwriting, delay in learning hours, days, weeks and months of the year, among many other problems usually related to abnormalities in the development of the parietal lobe and to an abnormal magnocellular system (Carlson, 2002). Supporting this hypothesis, the authors emphasize that many dyslexics complained that the letters appear to be moving continuously on the page or seem to be mixed, which would be justified by the fact that these individuals present a binocular instability and an unstable visual perception. The uncontrolled movements of their eyes are interpreted by these dyslexics the movement of the letters.

The studies of magnocellular neurons and the emphasis on this abnormal magnocellular processing bring future expectations for the early discovery of dyslexia. This would be by measuring the temporal visual perception of children at risk for dyslexia before literacy, leading to a better school support.

Many researchers in favor of the magnocellular theory not only discuss but support and defend other area for research, the nutrition field. According to these authors, nutrition may be related to the case of dyslexia, especially when it comes to visual deficits. According to proponents of "nutritional approach to dyslexia", visual deficits that may contribute to the developmental dyslexia may be based on abnormalities in the metabolism of polyunsaturated long chain fatty acids (Kajihara, 2008). These fatty acids are extremely important for the normal development of brain structures and functions. This deficiency impairs the shape of the phospholipids cell membranes and affects greatly the cell surface, as is the case of Magnocellular cells. Thus, compared to normal subjects, dyslexic individuals have a higher proportion of smaller Magnocellular cell probably due to the deficiency of these lipids that affect the growth of these neurons (Taylor et al., 2000).

In recent research, Cyhlarova et al. (2007) found no significant differences in the levels of fatty acids of cell membranes between dyslexic subjects and control group. However, there was an association between higher concentrations of omega- 3 and omega- 6 to the individuals with better performance in reading, suggesting that the balance omega- $3 / 6$ is particularly relevant for dyslexia.

The language involves several factors to produce the human communication system. The visual and auditory systems are involved in spoken and written discourse, thus research groups have focused in the auditory-neuro- 
logical disorders that may be correlated with the development of some learning problems, such as dyslexia. Research by Sauer et al. (2006) evaluated children with dyslexia with SPECT (Single Photon Emission Computed Tomography). This test provides information about blood flow and functional neurological deficits of the brain, which lead to the inability to achieve reading and writing. The research concluded that children with dyslexia show changes in the neurological processing center. The changes observed may indicate difficulties in the organization of sound events in time, impaired short-term auditory memory and difficulty in blocking competitive sounds. According to the authors, these results are compatible with those shown in the literature, pointing out another aspect that may be related to dyslexia which is being increasingly investigated. Thus, studies of the correlation between neural changes and dyslexia may be further scrutinized as future tests for the characterization of dyslexic children, encouraging early treatment and initial follow-up of educators, health professionals and family.

\subsection{Treatment and Prevention of Developmental Dyslexia}

The main goal of better understanding dyslexia is prevention. It is important to consider that if the children at risk for developing dyslexia are identified even before school or at least in the early stages of literacy, there is an opportunity to intervene therapeutically, minimizing and even eliminating gaps in reading learning and in the process of acquiring language (Gabrieli, 2009).

According to Gabrieli (2009), studies with kindergarten students reinforced the possibility of prevention and early treatment. This research focused on the observation of learning letter names and sounds made by children, in addition to phonological awareness and naming speed. When readers identified as a risk group for the development of dyslexia had stepped up, especially on the topics of learning described above, most children failed to produce an efficient reading in the average age for this activity. Thus, the observation of the students not only identifies possible future dyslexic readers as the feasibility of preventing major reading difficulties which, for over the years, become more difficult to correct.

It is possible to observe that is ever more necessary the popularization of dyslexia and other learning disorders not only for the academic people but mainly to the educational professionals so they may work together with the school, family and health professionals to monitor the development of this individual while avoiding the difficulties and constraints that these disorders bring to these children.

In general, neurophysiologic effective repair is associated with increased activation of frontal and temporalparietal regions. Importantly, dyslexics usually exhibit a decrease or absence of activation of these regions. Immediately after the intervention, hiperactivation of the right hemisphere is also observed, which may reflect a change in the interpretation of visual messages, such as letters and words, helping to improve the general interpretation of a text. Thus, individuals with dyslexia may receive interventions that increase activation of both left and right hemispheres. These changes in brain function can be maintained for some time after the intervention. However, it is unclear how these hiperactivation will be done, or what constancy of these activations and time will be needed for effective treatment of dyslexic individuals (Gabrieli, 2009).

Neuroimaging studies have not revealed what differences lead to a particular child respond better or worse to the treatment. This identification would be very useful as they could determine the most appropriate treatment, and intervention system (Gabrieli, 2009). However, several factors related to dyslexia as well as brain areas and activations or hipoactivations are still not very clear. This hinders the classification of the disorder and its variants, making it difficult to obtain positive results comparing treatments and prevention.

Dyslexia is also attributed to poor ocular dominance and visual processing deficits in cortical area and in the visual extra-striate central nucleus. This affects the identification and correlation of words and phrases. Thus, treatment guidelines related to the visual system involved in dyslexia suggest, for individuals with the disorder, eye exercises, tasks of eye-hand coordination and other exercises designed to improve the activity of visual memory and visual-motor dyslexic readers. Although this is a research line related to a potential factor for characterization of dyslexia, few studies report the relationship between intervention visual/ocular-motor with a concomitant improvement in reading skill (Gabrieli, 2007).

Currently, overlapping colored lenses (e.g. Glasses with lenses of different colors) are pointed as a therapy for various learning disorders related to reading. However, these studies also are not numerous and methodologically incomplete, which prevent the analyses of the effectiveness of this type of intervention (Gabrieli, 2007). A 
more recent review by the American Academy of Ophthalmology concluded that there is no scientific evidence that supports that behavior therapies involved in vision, vision therapy orthotics, or even overlapping colored lenses are effective treatments for learning disabilities. Therefore, current consensus considers that visual therapies are not an evidence-based treatment for reading or language-related disorders.

\section{Dyslexia in the School Context}

Dyslexia is not simply related to environmental factors, socioeconomic or disinterest from students, teachers and/or family. Instead, it is a disorder with a possible hereditary origin in which failed connections and pathways affect the development of speech and writing processes. Despite the technology involving brain scanning and the accessibility of information about this disorder on the internet, family members and especially educators know almost nothing about it.

Importantly, the educational professionals should know at least how to identify children with dyslexia so psychopedagogical measures can be taken. Neurological aspects relating to the prevention and treatment of dyslexia need special attention. However, in everyday life, dyslexia has been addressed within the learning difficulties that a typical student has. It becomes crucial a brief overview of this disorder in the school context which is currently presented through various demonstrations of cognitive deficits.

According to the literature, there are some signs that allow recognizing a dyslexic child (Torres \& Fernandes, 2001). It is important to emphasize that only the observation of these signs does not define or classify these dyslexic individuals, but serves as an indicator for exploring this possibility. The diagnosis and treatment of dyslexic individuals need professionals specialized in learning disabilities and speech therapists, psychologists, educators and physicians.

Pestun \& colleagues (2002) emphasize the need for this interdisciplinary team as reading involves neurophysiologic, psychological, socio-cultural, socioeconomic, and educational aspects, among others. According to these and other authors, the difficulties observed in reading and writing of a dyslexic child, including perceptual-motor disabilities, are similar to non-dyslexic children in a transient state, as they also go through the initial processes of learning.

Currently, the diagnosis of dyslexia is based on family history and clinical, psychometric tests, tests of phonological awareness, language, reading and spelling (Teles, 2004). Looking into different resources may increase the diagnosis capacity (e.g. genes x neuroimaging) (Eicher \& Gruen, 2013).

Some linguistic features observed in the school context may indicate a child at risk, including:

- Changes in the sequence of letters that form syllables and words;

- Confusion of letters opposing symmetry, such as b for $\mathrm{d}$ or $\mathrm{p}$;

- Confusion of letters with similar sounds;

- Difficulty in understanding what is being read;

- Difficulty understanding jokes, proverbs and slang;

- Difficulties in distinguishing between similar sounds such as p-b; t, f-v; m-n;

- Difficulty in learning little songs and rhymes;

- Difficulty with fine motor skill;

- Difficulty in pronunciation of phonemes;

- Difficulty in learning foreign languages;

- Errors in the separation of words;

- Problems with laterality (right and left);

- Omission of letters or syllables while writing;

- Reduced Vocabulary.

Dyslexic children find extreme difficulty in reading, and usually pay little attention to it, thus preventing the ability of reporting what was read. Another reason for an ineffective reading is the lack of pace, which commonly occurs with dyslexia, further complicating the interpretation. It also can pose difficulties in spelling, since the oral language and writing have a great relationship with each other and both are a challenge for dyslexics (Torres \& Fernandes, 2001; Capellini et al., 2007).

It is important to notice that dyslexic children are able to record images, extremely creative, understand very well the stories told and have a high ability to learn (Teles, 2004). However, in general, the dyslexic individuals are seen by parents, teachers and peers as less intelligent, and often end up being excluded from the group be- 
cause of lack of skills equal to those of another class and for not keeping a behavior adequate in most cases.

According to Amorim (2008) and Stefanini \& Cruz (2006), emotional problems related to educational issues are common to dyslexics. They are often treated as inferior because they do not meet the expectations of parents and teachers, who tend to require the child to be like the others. They are classified as irresponsible and incapable, which may reduce their willingness to go to school, helping indirectly in the evolution of the disorder.

Thus, it is important to recognize not only the difficulties, but also the skills of dyslexic students. Identify areas of the curriculum where the student presents a good performance may allow them to feel in the group (Amorim, 2008). Senna (2008) reported that these students cannot be included in "failed" category only because they do not meet expectations related to the learning of certain contents. The conduct of social-affective environment for the students in the school should become much more important than their performance on tests.

The dyslexics are part of the group of students with special needs. They present some specific need for getting proper education, which should be attended to avoid hindering their learning and development. For that matter, the educational professionals should have the proper training necessary to deal with the learning difficulties of these students, working within their capabilities and limitations. It is essential that the educator is qualified to help these children with the effective approach needed for the child proper development (Bueno, 1999; Amorim, 2008; Souza, 2008).

Ignoring dyslexia does not help to overcome it, however, contribute to its aggravation. This perspective reflects the lack of scientific knowledge about dyslexia on the teaching methods to be used and the benefits of early intervention and specialized (Teles, 2004). However, it is important to note that, on the other hand, many children have been categorized as dyslexic by the simple fact that they are following your class more slowly, without any medical foundation and theoretical. In some cases, the school blames the student for the school problems in teaching and educational processes.

According to Amorim (2008) and Souza (2008) the mission of educators includes:

a) Encourage speech, self-esteem and confidence of the student, appreciating small achievements, respecting the different issues presented by it;

b) Assist the student's daily life with simple strategies such as summaries of the programs to be developed, starting each new content with schemes as facilitators mental maps and summaries of key points, using audiovisual resources to support and introduce new vocabulary in context;

c) Propose activities that make interacting with colleagues, and if this does not occur naturally, increase the time limit for performing tasks;

d) Evaluate her/his orally, let them express verbally throughout the lesson, etc.

In other words, small steps that should be taken regardless of the presence of students with difficulties since, in general, these are measures that support a more robust learning by all students. Since a classroom displays dyslexic students or others with any other learning difficulties, and considering that each individual is unique and deserves to be treated as such, it is essential that the teacher uses tools for maximum development of each student.

\section{Final Considerations}

Individuals with developmental dyslexia have neurophysiologic, neuroanatomical, and possibly genetic differences, with no single factor involved, but a number of factors which together characterize this disorder and its various subdivisions. Thus, for concrete and effective treatments, it is necessary to continue these studies in an attempt to reach a "common denominator", or several of them, thus dyslexic individuals will be properly classified and then evaluated, treated and monitored.

One factor that seems to be as much consensus among the scientific and educational communities is that these children need early and specific monitoring to reduce the chances of developing specific reading difficulties in the schooling process.

\section{Acknowledgements}

We thank to FAPERJ, CAPES, and CNPq for the fellowships and financial support.

\section{References}

Amorim, R. E. (2008). Games, Toys and Pranks in the Development of Dyslexic Children. 2008. 37f. End of Course Work 
(Specialization in Broad Sense-Learning Disabilities). São Paulo: Reference Center for Learning Disorders.

Barton, J. J. et al. (2014). The Word-Length Effect in Reading: A Review. Cognitive Neuropsychology, 31, 378-412. http://dx.doi.org/10.1080/02643294.2014.895314

Bear, M. F. et al. (2008). Neuroscience: Uncovering the Nervous System (3rd ed.). São Paulo: Artmed.

Bishop, D. V. M. (2009). Genes, Cognition and Communication: Insights from Neurodevelopmental Disorders. Annals of the New York Academy of Sciences, 1156, 1-18. http://dx.doi.org/10.1111/j.1749-6632.2009.04419.x

Bueno, J. G. S. (1999). Children with Special Educational Needs, Educational Policy and the Teachers Formation: Generalists or Specialists. Revista Brasileira de Educação Especial, 3, 7-25.

http://www.educacaoonline.pro.br/index.php?option=com_content\&view=article\&id=67:criancas-com-necessidades-educ ativas-especiais-politica-educacional-e-a-formacao-deprofessores-generalistas-ou-especialistas \&catid=5:educacao-especia $\underline{1 \& \text { Itemid }=16}$

Capellini, S. A. et al. (2007). Performance of Good Readers, Dyslexic and with Attention Deficit Hyperactivity Disorder Students in Rapid Automatized Naming. Revista da Sociedade Brasileira de Fonoaudiologia, 12, 114-119.

Carlson, N. R. (2002). Behavioral Physiology (7th ed.). 1st Brazilian Edition, São Paulo: Manole.

Ciasca, S. M. (2004). Learning Disorders: Interdisciplinary Assessment Proposal (2nd ed.). São Paulo: Casa do Psicólogo. http://books.google.com.br/books?hl=ptBR\&lr=\&id=bKBjBR45omAC\&oi=fnd\&pg=PA19\&dq=Dist $\%$ C $3 \%$ BArbios + de + $\underline{\text { aprendiza- }}$

gem $+\mathrm{e}+\mathrm{de}+$ comportamento[\&ots=Y7xenlgNpn\&sig=kJYFibUUtUyh9lpcXAKNI2HGkhk\#v=onepage\&q=Dist $\%$ C3\%BA rbios $\% 20 \mathrm{de} \% 20$ aprendizagem $\% 20 \mathrm{e} \% 20 \mathrm{de} \% 20$ comportamento[ $\& \mathrm{f}=$ false

Corballis, M. C. (2014). Left Brain, Right Brain: Facts and Fantasies. PLoS Biology, 12, e1001767. http://dx.doi.org/10.1371/journal.pbio.1001767

Cyhlarova, E., Gordon Bell, J., MacKinlay, E. E. et al. (2007). Membrane Fatty Acids, Reading and Speeling in Dyslexic and Non-Dyslexic Adults. European Neuropsychopharmacology, 17, 116-121.

http://dx.doi.org/10.1016/j.euroneuro.2006.07.003

Eckert, M. A., Leonard, C. M., Richards, T. L. et al. (2003). Anatomical Correlates of Dyslexia: Frontal and Cerebellar Finds. Brain, 126, 482-494. http://dx.doi.org/10.1093/brain/awg026

Eicher, J. D., \& Gruen, J. R. (2013). Imaging-Genetics in Dyslexia: Connecting Risk Genetic Variants to Brain Neuroimaging and Ultimately to Reading Impairments. Molecular Genetics and Metabolism, 110, 201-212. http://dx.doi.org/10.1016/j.ymgme.2013.07.001

Eicher, J. D., Powers, N. R., Miller, L. L. et al. (2014). Characterization of the DYX2 Locus on Chromosome 6p22 with Reading Disability, Language Impairment, and IQ. Human Genetics, 133, 869-881. http://dx.doi.org/10.1007/s00439-014-1427-3

Etchepareborda, M. C., \& Habib, M. (2001). Neurobiological Basis of Phonological Awareness: Its Commitment to Dyslexia. Revista de Neurologia Clinica, 2, 5-23.

Facoetti, A., Turatto, M., Lorusso, M. L. et al. (2001). Orienting of Visual Attention in Dyslexia: Evidence for Asymmetric Hemispheric Control of Attention. Experimental Brain Research, 138, 46-53. http://dx.doi.org/10.1007/s002210100700

Gabrieli, J. D. E. (2009). Dyslexia: A New Synergy between Education and Cognitive Neuroscience. Science, 325, $280-283$. http://dx.doi.org/10.1126/science.1171999

Kajihara, O. T. (2008). Current Theoretical Models of Developmental Dyslexia. Olhar de Professor, 11, n. 001.

Kandel, E. et al. (2002). Principles of Neuroscience (4th ed.). São Paulo: Editora Manole.

Krafnick, A. J. et al. (2014). An Investigation into the Origin of Anatomical Differences in Dyslexia. Journal of Neuroscience, 34, 901-908.

Lent, R. (2004). One Hundred Billion Neurons: Fundamental Concepts of Neuroscience. São Paulo: Atheneu.

Leonard, C. M., \& Eckert, M. A. (2008). Asymmetry and Dyslexia. Developmental Neuropsychology, 33, 663-681. http://dx.doi.org/10.1080/87565640802418597

Leong. V., \& Goswami, U. (2014). Assessment of Rhythmic Entrainment at Multiple Timescales in Dyslexia: Evidence for Disruption to Syllable Timing. Hearing Research, 308, 141-161. http://dx.doi.org/10.1016/j.heares.2013.07.015

Luz, M. A. P. C. (2008). Dyslexia-Specific Difficulty in Language Processes. http://www.abpp.com.br/artigos/113.pdf

Machado, C. et al. (2006). Neuropsychology Evaluation of Dyslexia. Actas da XI Conferência Internacional de Avaliação Psicológica: Formas e Contextos. Braga: Universidade do Minho. Editor: Psiquilíbrios.

Marsh, R., Gerber, A. J., \& Peterson, B. S. (2009). Neuroimaging Studies of Normal Brain Development and Their Relevance for Understanding Childhood Neuropsychiatric Disorders. Journal of the American Academy of Child \& Adolescent 
Psychiatry, 47, 1233-1251. http://dx.doi.org/10.1097/CHI.0b013e318185e703

Perea, M., \& Panadero, V. (2014). Does Violin Activate Violin More than Viocin? On the Use of Visual Cues during VisualWord Recognition. Experimental Psychology, 61, 23-29. http://dx.doi.org/10.1027/1618-3169/a000223

Pestun, M. S. V., Ciasca, S., \& Gonçalves, V. M. G. (2002). The Importance of Interdisciplinary Staff in the Diagnosis of Developmental Dyslexia. Arquivos de Neuro-Psiquiatria, 60, 328-332. http://dx.doi.org/10.1590/S0004-282X2002000200029

Salles, J. F. et al. (2004). The Developmental Dyslexias: Neuropsychological and Cognitive Aspects (Vol. 10, pp. 109-132). São Paulo: Interações.

Sauer, L., Pereira, L. D., Ciasca, S. M. et al. (2006). Auditory Processing and SPECT in Children with Dyslexia. Arquivos de Neuro-Psiquiatria, 64, 108-111. http://dx.doi.org/10.1590/S0004-282X2006000100022

Schumacher, J., Hoffmann, P., Schmäl, C. et al. (2007). Genetics of Dyslexia: The Evolving Landscape. Journal of Medical Genetics, 44, 289-297. http://dx.doi.org/10.1136/jmg.2006.046516

Senna, L. A. G. (2008). Teacher Training and Inclusive Education (Vol. 38). São Paulo: Cadernos de Pesquisa.

Simpson, N. H., Addis, L., Brandler, W. M. et al. (2014). Increased Prevalence of Sex Chromosome Aneuploidies in Specific Language Impairment and Dyslexia. Developmental Medicine \& Child Neurology, 56, 346-353. http://dx.doi.org/10.1111/dmcn.12294

Souza, E. C. (2009). The Dyslexic as a Subject of Teaching-Learning Process. Contrapontos (UNIVALI), 8, 423-432.

Stefanini, M. C., \& Cruz, S. A. (2006). Learning Disabilities and Its Causes: The Teacher of 1st to 4th Grades of Elementary School Looking. Educação, Porto Alegre-RS, ano XXIX, n. 1, p. 85-105, jan-abr.

Stein, J., \& Walsh, V. (1997). To See but Not to Read; the Magnocellular Theory of Dyslexia. Trends in Neurosciences, 20 , 147-152. http://dx.doi.org/10.1016/S0166-2236(96)01005-3

Svidnicki, M. C. C. M., Salgado, C. A., Lima, R. F. et al. (2013). Study of Candidate Genes for Dyslexia in Brazilian Individuals. Genetics and Molecular Research, 12, 5356-5364. http://dx.doi.org/10.4238/2013.November.7.10

Tailby, C., Weintrob, D. L., Saling, M. M. et al. (2014). Reading Difficulty Is Associated with Failure to Lateralize Temporooccipital Function. Epilepsia, 55, 746-753. http://dx.doi.org/10.1111/epi.12607

Talcott, J. B. et al. (1998). Visual Magnocellular Impairment in Adult Developmental Dyslexics. Neuro-Ophthalmology, 20, $1-22$.

Taylor, K. E. et al. (2000). Dyslexia in Adults Is Associated with Clinical Signs of Fatty Acid Deficiency. Prostaglandins, Leukotrienes and Essential Fatty Acids, 63, 75-78. http://dx.doi.org/10.1054/plef.2000.0195

Teles, P. (2004). Dyslexia How to Identify? How to Intervene? (Vol. 20). Portugal: Revista Portuguesa de Clínica Geral. nov./dez.

Temple, E., Poldrack, R. A., Salidis, J. et al. (2001). Disrupted Neural Responses to Phonological and Orthographic Processing in Dyslexic Children: An fMRI Study. Neuroreport, 12, 299-307. http://dx.doi.org/10.1097/00001756-200102120-00024

Torres, R. M. R., \& Fernández, P. F. (2001). Dyslexia, Dysgraphia and Dysorthographia. Portugal: McGraw-Hill.

Turkeltaub, P. E., Goldberg, E. M., Postman-Caucheteux, W. A. et al. (2014). Alexia due to Ischemic Stroke of the Visual Word form Area. Neurocase, 20, 230-235. http://dx.doi.org/10.1080/13554794.2013.770873

Vygotsky, L. (2008). Thought and Language. São Paulo: Martins Fontes Editora.

Williams, J., \& O’Donovan, M. C. (2006). The Genetics of Developmental Dyslexia. European Journal of Human Genetics, 14, 681-689. http://dx.doi.org/10.1038/sj.ejhg.5201575

Zdrojewska, J., \& Coffey, E. T. (2014) The Impact of JNK on Neuronal Migration. Advances in Experimental Medicine and Biology, 800, 37-57. http://dx.doi.org/10.1007/978-94-007-7687-6 3 